\title{
Quantum Mechanical / Molecular Mechanical Self-Consistent Madelung Potential Method for Treatment of Polar Molecular Crystals
}

\author{
GYÖRGY G. FERENCZY,,$^{1,2}$ GÁBOR I. CSONKA, ${ }^{3}$ \\ GÁBOR NÁRAY-SZABÓ, ${ }^{4}$ JÁNOS G. ÁNGYÁN ${ }^{5}$ \\ ${ }^{1}$ Chemical Works of Gedeon Richter Ltd., P.O. Box 27, H-1475 Budapest, Hungary \\ ${ }^{2}$ Department of Chemical Information Technology, Technical University of Budapest, Szent Gellért tér \\ 4, H-1111 Budapest, Hungary \\ ${ }^{3}$ Department of Inorganic Chemistry, Technical University of Budapest, H-1521 Budapest, Hungary \\ ${ }^{4}$ Department of Theoretical Chemistry, Eötvös University Budapest, P.O. Box 32, H-1518 Budapest \\ 112, Hungary \\ ${ }^{5}$ Laboratoire de Chimie Théorique, Université Henri Poincaré, CNRS URA 510, B.P. 239, F-54506 \\ Vandoèuvre-lès-Nancy Cedex, France
}

Received 28 July 1996; accepted 20 July 1997

\begin{abstract}
The self-consistent Madelung potential (SCMP) approach for calculating molecular wave functions for a subunit embedded in a symmetrical environment constituted by the copies of the subunit is implemented with semiempirical NDDO model Hamiltonians and supplemented with empirically parameterized dispersion-repulsion interaction potentials. This model yields sublimation enthalpies in good agreement with available experimental data for a series of molecular crystals, including imidazol, benzimidazole, urea, urethane, dicyaneamide, formamide, uracil, cytosine, maleic anhydride, succinic anhydride, and 1,3,5-triamino-2,4,6-trinitro-benzene. The SCMP-NDDO method, which avoids difficulties concerning the parametrization of charges in the molecular mechanics force fields, is proposed mainly for the treatment of molecular crystals with large unit cells. It might be particularly useful where
\end{abstract}

Correspondence to: Dr. J. G. Ángyán; e-mail: angyan@lctn.unancy.fr

Contract/grant sponsor: Balaton; contract/grant number 52

Contract/grant sponsor: National Fund for Scientific Research, Hungary; contract/grant numbers OTKA/T19387 and OTKA/T22012 
important charge reorganization is expected under the effect of the crystal field. Charge distributions, obtained by the SCMP and the simple dielectric cavity self-consistent reaction field models, are compared and analyzed. (C) 1998 John Wiley \& Sons, Inc. J Comput Chem 19: 38-50, 1998

Keywords: quantum mechanics; molecular mechanics; self-consistent Madelung potential; polar molecular crystals

\section{Introduction}

( uantum chemical treatment of organic molecular crystals constitutes a major challenge of molecular modeling. Empirical force field methods often correctly describe the crystal structure, ${ }^{1}$ but such classical approaches have recently been recognized as missing certain structure-defining interactions. ${ }^{2}$

Apolar or moderately polar systems could be successfully characterized by force fields without $r^{-1}$-dependent explicit Coulomb terms, ${ }^{1,3}$ but consideration of the strong electrostatic forces seems to be a necessity in strongly polar or partly ionic systems. ${ }^{4-7}$ The introduction of explicit Coulomb terms leads to a serious complication in the parameterization of the force fields. While the nonelectrostatic van der Waals parameters of a given atom type are transferable from one system to another, atomic charges, entering in the Coulomb part of the force field, show very limited transferability. In a series of closely related molecules charge transferability may be relatively poor, ${ }^{8-10}$ and even the same molecule changes its charge distribution as a function of its conformation. ${ }^{11-16}$ Furthermore, the chemical environment may lead to substantial reorganization of the charge density with respect to the isolated molecule, which makes questionable the usefulness of atomic charges determined in vacuo. ${ }^{17}$ Such effects might be exalted in the solid phase. For example, in-crystal dipole moments can be considerably enhanced. ${ }^{18}$ One can cite the example of 2-methyl-4-nitroaniline, where experimental evidence seems to indicate the doubling of the free-molecule dipole moment from $9 \mathrm{D}$ to $20 \mathrm{D}$, due to crystal field effects. ${ }^{19}$ Even if this estimate of the dipole moment enhancement may be somewhat exaggerated due to difficulties in assigning experimental charge density to individual molecules in the crystal, it is quite clear that in such cases the electronic polarization is strongly nonlinear. Under the effect of the crystal field, strongly polar proton transferred structures may be stabilized (e.g., amino acid crystals ${ }^{4}$ ) and even intermolecular charge transfer may take place, as in donor-acceptor complex crystals. ${ }^{20}$ Because the amplitude of these charge density rearrangements exceeds the linear regime, the usual polarizable models are insufficient and consideration of higher hyperpolarizabilities would be necessary. The most satisfactory way to tackle this problem consists of the self-consistent calculation of the charge distribution of the subunits in each new situation encountered in a solid environment.

A straightforward method to avoid the difficulties related to the parameterization of intermolecular interactions in a solid is to explicitly calculate the electronic structure, for example, by the periodic Hartree-Fock (HF) approach. ${ }^{21}$ However, in this method relatively few electrons per unit cell can be treated and the absence of electron correlation from the model (missing dispersion interactions) results in an incomplete description of the intermolecular forces. Recent developments in density functional-based approaches, using a plane wave basis and ultrasoft pseudopotentials, may constitute a promising alternative $\mathrm{e}^{22-24}$ for molecular crystals as well, as witnessed by the first attempts along this line. ${ }^{25}$

Another possibility is to build finite clusters of molecules, modeling the most important interactions of a central molecule with its neighbors. ${ }^{26}$ This procedure is quite well adapted to study crystal growth, ${ }^{27}$ but it is less appropriate for the simulation of real solids where the 3-dimensional periodicity should be properly taken into account. Large $a b$ initio models, comprising at least the nearest neighbors of a central molecule, embedded in the field of point charges is a relatively expensive, but successful way of including most of the important intra- and intermolecular interactions in the computational model. ${ }^{28-31}$ The nearest neighbors can be represented more accurately by $a b$ initio model potentials: this procedure has been used mainly for ionic systems. ${ }^{32-35}$

The self-consistent Madelung potential (SCMP) approach $^{36,37}$ seems to be a good compromise to 
treat local electronic structures with traditional quantum chemical methods and simultaneously to take into consideration long-range electrostatic and induction effects in a solid. This approach can be considered as a kind of electrostatic embedding, where explicit quantum chemical calculation is performed only on a basic motif (unit cell or asymmetric unit), and electrostatic interactions with the copies of this basic unit are taken into account by modifying the electronic Hamilton operator. Because the charge distribution of the neighbors is determined by that of the basic motif by the virtue of symmetry, the calculation should be repeated until self-consistency in the perturbing potential. Various implementations of the SCMP model, mainly at the $a b$ initio level, have been published during the past 25 years. ${ }^{38-42}$ Although molecular crystals with relatively large unit cells can be treated by $a b$ initio versions of SCMP-like methods, we feel that a semiempirical version, which allows rapid calculation on unit cells constituted of about $30-60$ atoms or more, is in order.

The present implementation of the SCMP approach, integrated with a simple dispersion-repulsion potential energy function, should be considered as a kind of mixed quantum mechanical/ molecular mechanics (QM/MM) model specifically adapted to the solid phase. The reliability of the combined energy expression (intramolecular energy and intermolecular electrostatic and induction effects are taken into account at the semiempirical NDDO level, and intermolecular dispersion and repulsion are included by the empirical potential) has been tested on dimers. In these dimer studies we used the fact that the presence of the translational symmetry does not make it obligatory to use an SCMP-like approach: it can be applied to $n$-mers of identical subunits related by any kind of symmetry operation. Only half of the dimer is considered explicitly and the charge distribution of the second half is generated by the appropriate symmetry operation (e.g., inversion center, mirror plane, etc.). This situation is analyzed in some detail in the following.

Results for the sublimation heat of a few selected crystals, where reliable experimental data were available, were compared. Recently it became a common practice to mimic a solid crystalline environment by a simple continuum dielectric model, ${ }^{43}$ like in solvent effect theories. We examined whether the SCMP enhancement of the dipole moment can be reproduced by a continuum dielec- tric model using an ellipsoidal cavity. This analysis is also presented after the discussion of the energetic results on the sublimation heats.

\section{Method}

Mixed QM/MM models have an increasingly important role in the treatment of extended systems, primarily in the study of biopolymers and their reactions (see refs. 44-46). The philosophy of these methods is that the total system is separated into a subsystem and an environment: the former is treated quantum chemically, while the latter is treated classically. Our approach is similar in the sense that the crystal is separated into a quantum chemical motif and the rest of the system. The motif is defined as the portion of the system from which the charge distribution of the whole crystal can be built up by symmetry operations. Although the most straightforward choice for the motif is the asymmetric unit, any multiple of the asymmetric unit might be appropriate to generate the full crystal.

The motif is described by a standard quantum chemical method, in our case by a semiempirical model Hamiltonian. The environment is represented by a set of point multipoles whose effect is included in the quantum chemical Hamiltonian. Because the environment is built up from repeated motifs, the point multipoles representing the environment have to be consistent with the wave function of the quantum motif. The interaction of the motif with its environment is complemented with a classical atom-atom dispersion-repulsion potential.

The quantum chemical part of our model is based on the SCMP approach discussed in detail in refs. 37 and 47. Here the main features of the method are recapitulated and the semiempirical parametrization of the QM and MM parts of the Hamiltonian are described. The behavior of the mixed QM/MM energy is discussed in terms of physical contributions and analyzed numerically with the example of symmetrical dimers.

\section{SCMP APPROACH}

The molecular crystal is considered to be a system of interacting subsystems (motifs). A unit cell is constituted from $Z$ motifs, which are usually identical with a crystallographic asymmetric unit. The total energy per asymmetric unit can be 
expressed as the sum of the quantum chemical total energy of the arbitrarily selected $i$ th motif (asymmetric unit) of the $L=0 \equiv(0,0,0)$ unit cell and two interaction terms, namely the QM treated electrostatic and the MM dispersion-repulsion contribution:

$$
\begin{aligned}
E^{\mathrm{SCMP}}= & \left\langle\Psi^{i, 0}\left|\hat{H}_{0}^{i}\right| \Psi^{i, 0}\right\rangle \\
& +\frac{1}{2} \sum_{j}^{Z} \sum_{L}^{\infty}\left\langle\Psi^{i, 0}\left|\hat{\varrho}_{\mathbf{r}}^{i}\right| \Psi^{i, 0}\right\rangle T_{\mathbf{r r}^{\prime}}\left\langle\Psi^{j, L}\left|\hat{\varrho}_{\mathbf{r}^{i}}\right| \Psi^{j, L}\right\rangle \\
& +\frac{1}{2} \sum_{j}^{Z} \sum_{L}^{\infty} E_{i 0, j L}^{\mathrm{MM}} .
\end{aligned}
$$

In this expression $\Psi^{j, L}$ is the wave function of the motif $j$ in the unit cell labeled by a set of three integers, $L=\left(l_{1}, l_{2}, l_{3}\right) ; \hat{H}_{0}^{i}$ is the Hamiltonian of the isolated motif $i$; $\hat{\varrho}_{\mathrm{r}}^{i}$ is the operator of the total charge density of the $i$ th motif,

$$
\hat{\varrho}_{\mathbf{r}}^{i}=\sum_{A \in i} Z_{A} \delta\left(\mathbf{r}-\mathbf{R}_{A}\right)-\sum_{\alpha \in i} \delta\left(\mathbf{r}-\mathbf{r}_{\alpha}\right),
$$

where the nuclei $A$ have the charge $Z_{A}$ and the electrons $\alpha$ belong to the motif; $T_{\mathrm{rr}^{\prime}}=\left|\mathbf{r}-\mathbf{r}^{\prime}\right|^{-1}$ is the Coulomb-kernel; and $E_{i 0, j L}^{\mathrm{MM}}$ is an empirical potential energy function that takes into account those interactions between motifs $i 0$ and $j L$ that are missing from the quantum chemical energy expression. The repeated lower indices $\mathbf{r}$ in the second term imply a generalized Einstein convention: one should integrate over the corresponding space variables to obtain the Coulomb interaction energy. The summations run over the $Z$ symmetry operations, $j$, within the unit cells. The primed sums running over the unit cells $L$ exclude the $L=(0,0,0)$ term if $i=j$.

The hypothesis underlying this energy expression is that the total wave function of the crystal is a Hartree product of normalized, antisymmetrized subsystem wave functions, which are strongly orthogonal to each other. We can use the fact that the wave function $\Psi^{j, L}$ located in an arbitrary unit cell can be generated from the wave function $\Psi \equiv \Psi^{1,0}$ of the motif by an appropriate rototranslation, $\hat{\mathbf{Q}}^{j, L}$,

$$
\Psi^{j, L}=\hat{\mathbf{Q}}^{j, L} \boldsymbol{\Psi} \quad \hat{Q}^{j, L}=\left\{\hat{\mathbf{U}}_{j} \mid \mathbf{t}_{j}+\mathbf{R}_{L}\right\},
$$

where $\hat{\mathbf{U}}_{j}$ is a rotation, $\mathbf{t}_{j}$ is a translation within the unit cell, and $\mathbf{R}_{L}=l_{1} \mathbf{a}=l_{2} \mathbf{b}+l_{3} \mathbf{c}$ is a lattice translation defined in terms of the integers $\left(l_{1}, l_{2}, l_{3}\right)$ and the lattice vectors, $\{\mathbf{a}, \mathbf{b}, \mathbf{c}\}$. (For the sake of simplicity we assumed that all the motifs of the unit cell are identical; generalization to binary, etc. systems is straightforward.) By virtue of the crystal space group symmetry, the SCMP energy depends only on the wave function of the asymmetric unit:

$$
\begin{aligned}
E^{\mathrm{SCMP}}= & \left\langle\Psi\left|\hat{H}_{0}\right| \Psi\right\rangle+\frac{1}{2}\left\langle\Psi\left|\hat{\varrho}_{\mathbf{r}}\right| \Psi\right\rangle G_{\mathrm{rr}^{\prime}}^{\mathrm{MAD}}\left\langle\Psi\left|\hat{\varrho} \mathbf{r}^{\prime}\right| \Psi\right\rangle \\
& +\frac{1}{2} \sum_{j}^{Z} \sum_{L}^{\infty} E_{10, j L}^{\mathrm{MM}} .
\end{aligned}
$$

Here the symmetrical Madelung kernel, $G_{\mathbf{r r}^{\prime}}^{\mathrm{MAD}}$,

$$
\begin{aligned}
G_{\mathrm{rr}}^{\mathrm{MAD}} & =\sum_{j}^{Z} \sum_{L}^{\infty} T\left(\mathbf{r}, \hat{\mathbf{Q}}^{j, L} \mathbf{r}^{\prime}\right) \\
& =\sum_{j}^{Z} \sum_{L}^{\infty}\left|\hat{\mathbf{U}}_{j} \mathbf{r}^{\prime}-\mathbf{r}+\mathbf{t}_{j}+\mathbf{R}_{L}\right|^{-1},
\end{aligned}
$$

gathers the lattice sums over sublattices.

Assuming that the MM dispersion-repulsion energy does not depend on the motif wave functions, the energy $E^{\mathrm{SCMP}}$ is made stationary by the solution of the following nonlinear Schrödinger equation:

$$
\left(\hat{H}_{0}+\hat{\rho}_{\mathrm{r}} G_{\mathrm{rr}^{\prime}}^{\mathrm{MAD}}\left\langle\Psi\left|\hat{\rho}_{\mathrm{r}^{\prime}}\right| \Psi\right\rangle\right)|\Psi\rangle=\mathscr{E}|\Psi\rangle .
$$

This nonlinear Schrödinger equation is quite similar to the self-consistent reaction field (SCRF) equations ${ }^{48}$ of solvent effects theories. The main difference is that the reaction potential response function of the solvent (expressed usually by the reaction field factors) is replaced here by the Madelung interaction kernel, $G_{\mathrm{rr}^{\prime}}^{\mathrm{MAD}}$.

The solution of the nonlinear SCMP Schrödinger equation, eq. (5), can be obtained by any standard quantum chemical approach. For example, in the case of the HF-Roothaan (HFR) method the usual matrix elements $F_{\mu \nu}^{0}$ of the Fock operator should be completed by integrals involving the Madelung kernel:

$$
\begin{aligned}
F_{\mu \nu}= & F_{\mu \nu}^{0}+\sum_{\lambda \sigma} P_{\sigma \lambda}\left(\mu \nu\left|G_{\mathbf{r r}}^{\mathrm{MAD}}\right| \lambda \sigma\right) \\
& -\sum_{A}\left(\mu\left|Z_{A} G^{\mathrm{MAD}}\left(\mathbf{r}, \mathbf{R}_{A}\right)\right| \nu\right) .
\end{aligned}
$$

Here $Z_{A}$ is the nuclear charge, $P_{\nu \mu}=2 \sum_{a}^{\mathrm{occ}} c_{\mu a} c_{\nu a}$ is the density matrix, and the Coulomb-like two-electron Madelung integrals over atomic orbitals are defined as

$$
\begin{aligned}
& \left(\mu \nu\left|G_{\mathrm{rr}}^{\mathrm{MAD}}\right| \lambda \sigma\right) \\
& \quad=\iint d^{3} \mathbf{r} d^{3} \mathbf{r}^{\prime} \mu(\mathbf{r}) \nu(\mathbf{r}) G_{\mathbf{r r}^{\prime}}^{\mathrm{MAD}} \lambda\left(\mathbf{r}^{\prime}\right) \sigma\left(\mathbf{r}^{\prime}\right) .
\end{aligned}
$$


The SCMP-HFR equations can be solved either directly by updating the Madelung contribution to the Fock matrix element in each iteration, or indirectly by solving the HFR equations each time at a fixed value of the Madelung potential,

$$
V_{\mathrm{r}}^{\mathrm{MAD}}(\Psi)=G_{\mathrm{rr}^{\prime}}^{\mathrm{MAD}}\left\langle\Psi\left|\hat{\varrho}_{\mathrm{r}}\right| \Psi\right\rangle,
$$

and iterating until the Madelung potential converges.

The total energy per asymmetric unit is given as

$$
\begin{aligned}
E^{\mathrm{SCMP}}= & \frac{1}{2} \sum_{\mu \nu} P_{\mu \nu}\left(F_{\mu \nu}^{0}+h_{\mu \nu}^{0}\right) \\
& +\frac{1}{2} \sum_{A} \sum_{B} Z_{A} Z_{B} T\left(\mathbf{R}_{A}, \mathbf{R}_{B}\right) \\
& -\sum_{\mu \nu} P_{\mu \nu} \sum_{A}\left(\mu\left|Z_{A} G^{\mathrm{MAD}}\left(\mathbf{r}, \mathbf{R}_{B}\right)\right| \nu\right) \\
& +\frac{1}{2} \sum_{\mu \nu} \sum_{\lambda \sigma} P_{\mu \nu} P_{\lambda \sigma}\left(\mu \nu\left|G_{\mathrm{rr}^{\prime}}^{\mathrm{MAD}}\right| \lambda \sigma\right) \\
& +\frac{1}{2} \sum_{A} \sum_{B} Z_{A} Z_{B} G^{\mathrm{MAD}}\left(\mathbf{R}_{A}, \mathbf{R}_{B}\right) \\
& +\frac{1}{2} \sum_{j} \sum_{L}^{\infty} E_{10, j L}^{M M} .
\end{aligned}
$$

The actual implementation of the SCRF approach sould be completed by defining the parametrization of the quantum chemical Hamiltonian and the MM energy expression. These points are discussed in the following subsections.

\section{PARAMETERIZATION OF QUANTUM CHEMICAL HAMILTONIAN}

The present semiempirical implementation of the SCMP method is based on the NDDO model Hamiltonian of the motif. Among the most popular $\mathrm{MNDO}^{49}{ }^{4 M 1}{ }^{50}$ and $\mathrm{PM}^{51}$ models finally the AM1 parametrization has been retained (vide infra).

Choosing a representation for the electrostatic perturbation term remains, which couples the motif with its periodic images. A common feature in the NDDO models is to evaluate the intramolecular Coulomb integrals as damped interactions of point charge sets that mimic atomic multipole charge distributions. ${ }^{49}$ The generalization of this approximation to the SCMP Hamiltonian would be simple: the Madelung kernels should be calculated for all pairs of point charge centers and the matrix elements corrected by the core repulsion function taken for the nearest neighbors. Although such an approach has conceptual advantages, the core repulsion function is not always adapted to the description of intermolecular complexes. Therefore, an alternative solution has been chosen, where one replaces the point charge sets by the corresponding point multipoles. This leads to an equivalent long-range behavior of the electrostatic interactions, while the intermolecular repulsion energy (as well as other short-range contributions) can be taken care of more appropriately by the MM potential function.

The calculation of the electrostatic perturbation of the motif HF equations is based on the evaluation of the lattice sums. Up to the tensors of $R^{-3}$ the Ewald method was used, and the convergence parameters were determined by the method of Catti. ${ }^{52}$ Higher order tensors were calculated as direct space sums. The highest order tensor is that of $R^{-5}$, corresponding to the interaction between the products of two $p$ orbitals (i.e., between two quadrupoles).

An essential feature of the SCMP method is that the wave function of the motif is relaxed in the Madelung potential of the crystal. This means that, in addition to the electrostatic energy, which is usually defined as the Coulomb interaction of the in vacuo charge distribution of the motifs, induction effects are also included in the quantum chemical calculations.

\section{PARAMETRIZATION OF DISPERSION-REPULSION POTENTIAL}

To correctly describe the energetics (e.g., the sublimation energies) in molecular crystals, the further physical contributions to the total energy, like the long-range dispersion and the short-range penetration and overlap repulsion energies, should also be taken into consideration. These missing terms can be evaluated relatively easily by complementing the SCMP energy expression with an empirical dispersion-repulsion potential.

The 6-exp atom-atom model potential, derived by Filippini and Gavezzotti ${ }^{3,53}$ and modified by Willock et al., ${ }^{6}$ was selected because it has been applied successfully for molecular crystals. ${ }^{6}$ Its analytical expression,

$$
\begin{aligned}
E_{a b}^{\mathrm{MM}}= & \frac{\epsilon_{a b}}{(\lambda-6)} \\
& \times\left[6 \exp (\lambda) \exp \left(-\lambda \frac{R_{a b}}{R_{a b}^{0}}\right)-\lambda\left(\frac{R_{a b}^{0}}{R_{a b}}\right)^{6}\right],
\end{aligned}
$$


seems to be well adapted to describing the missing interaction terms. Here the parameters $\epsilon_{a b}=$ $\sqrt{\epsilon_{a a} \epsilon_{b b}}$ and $R_{a b}^{0}=\frac{1}{2}\left(R_{a a}^{0}+R_{b b}^{0}\right)$ are derived by using the common combining rules, and $\lambda$ is a universal parameter for all kinds of interactions. The total dispersion-repulsion energy between a pair of motifs is calculated as

$$
E_{i 0, j L}^{\mathrm{MM}}=\sum_{a \in i 0} \sum_{b \in j L} E_{a b}^{\mathrm{MM}}
$$

where $a$ runs over the atoms of the central motif and $b$ runs over atoms of $j L$, provided they are within a given cutoff distance. The cutoff radius, which varied between 40 and $60 \AA$ for the studied systems, was determined for each case such that its further increase affected the energy less than 0.1 $\mathrm{kJ} / \mathrm{mol}$.

\section{Analysis of SCMP Potential Energy}

\section{COMPONENTS OF SCMP LATTICE ENERGY}

The cohesion (lattice) energy of a crystal, $\Delta E^{\mathrm{SCMP}}$, is defined as the difference of the total SCMP energy and the isolated monomer energy, calculated from the in vacuo monomer wave function, $\Phi$ :

$$
\Delta E^{\mathrm{SCMP}}=E^{\mathrm{SCMP}}-\left\langle\Phi\left|\hat{H}_{0}\right| \Phi\right\rangle .
$$

As usual in the theory of intermolecular interactions, the total lattice energy can be decomposed to various physical contributions as

$$
\Delta E^{\mathrm{SCMP}}=E^{\mathrm{ele}}+E^{\mathrm{pen}}+E^{\mathrm{rep}}+E^{\text {ind }}+E^{\mathrm{dis}}+E^{\mathrm{cht}},
$$

namely, the first-order multipolar electrostatic, penetration, and overlap repulsion, as well as the second-order induction, dispersion, and charge transfer energies. Among these terms the multipolar electrostatic, $E^{\text {ele }}$, and induction, $E^{\text {ind }}$, can be easily identified from the quantum chemical calculation. The electrostatic energy, which is the Coulomb interaction of the unperturbed monomer charge distributions,

$$
E^{\mathrm{ele}}=\frac{1}{2}\left\langle\Phi\left|\hat{\varrho}_{\mathrm{r}}\right| \Phi\right\rangle G_{\mathrm{rr}^{\prime}}^{\mathrm{MAD}}\left\langle\Phi\left|\hat{\varrho}_{\mathrm{r}^{\prime}}\right| \Phi\right\rangle,
$$

can be obtained from the first SCMP iteration if the in vacuo density matrix is used as the initial guess. The induction energy, which is the energy gain due to the electric polarization of the motif charge densities,

$$
\begin{aligned}
E^{\text {ind }}= & \left\langle\Psi\left|\hat{H}_{0}\right| \Psi\right\rangle-\left\langle\Phi\left|\hat{H}_{0}\right| \Phi\right\rangle \\
& +\frac{1}{2}\left\langle\Psi\left|\hat{\varrho}_{\mathrm{r}}\right| \Psi\right\rangle G_{\mathrm{rr}^{\prime}}^{\mathrm{MAD}}\left\langle\Psi\left|\hat{\varrho}_{\mathrm{r}^{\prime}}\right| \Psi\right\rangle-E^{\text {ele }},
\end{aligned}
$$

is calculated from the converged charge density and includes the energy loss related to the deformation of the motif wave function. The long-range electrostatic + induction energy is expected to be reliable, because semiempirical NDDO methods yield quite reasonable charge distributions. Indeed, NDDO methods reproduce electrostatic effects correctly outside the van der Waals envelope of molecules. ${ }^{54}$

The remaining physical contributions, which are also expected to contribute significantly to the cohesion energy in crystals, are included in $E^{\mathrm{MM}}$. Penetration energy, $E^{\text {pen }}$, related to the nonmultipolar contribution to the electrostatic energy, is expected to be important for near atoms in the crystal. It is supposed to be included in the shortrange exponential component of the classical potential, along with the overlap repulsion energy, $E^{\text {rep }}$. The other essential component of $E^{\mathrm{MM}}$ is the dispersion energy, $E^{\text {dis }}$. We note that it cannot be obtained in the HF approximation; therefore, in the case of motifs composed of several molecules (this did not occur in the present study), the consideration of intramotif dispersion energy might be necessary.

\section{POTENTIAL CURVES OF SYMMETRICAL DIMERS}

The quantitative behavior of the potential energy model deserves further investigation. We think that knowledge of the variation of the potential with intermolecular distances is fundamental in exploring the range of the applicability of the model, and its correct behavior is a prerequisite for its applicability in future geometry optimization ${ }^{55}$ applications and in molecular dynamics studies.

The intermolecular potential of symmetrical dimers of $\mathrm{H}_{2} \mathrm{O}, \mathrm{HCOOH}, \mathrm{NH}_{2} \mathrm{OH}, \mathrm{CO}_{2}$, and $\mathrm{HCN}$ were calculated. These dimer structures do not necessarily correspond to the optimal orientation of the monomers; they were selected such that the two monomers were connected by a point group operation (inversion). In all five cases three intermolecular potentials were compared:

1. $\Delta E^{\mathrm{SCMP}}$ (calculation performed on a monomer and corrected by the symmetry-related partner), 

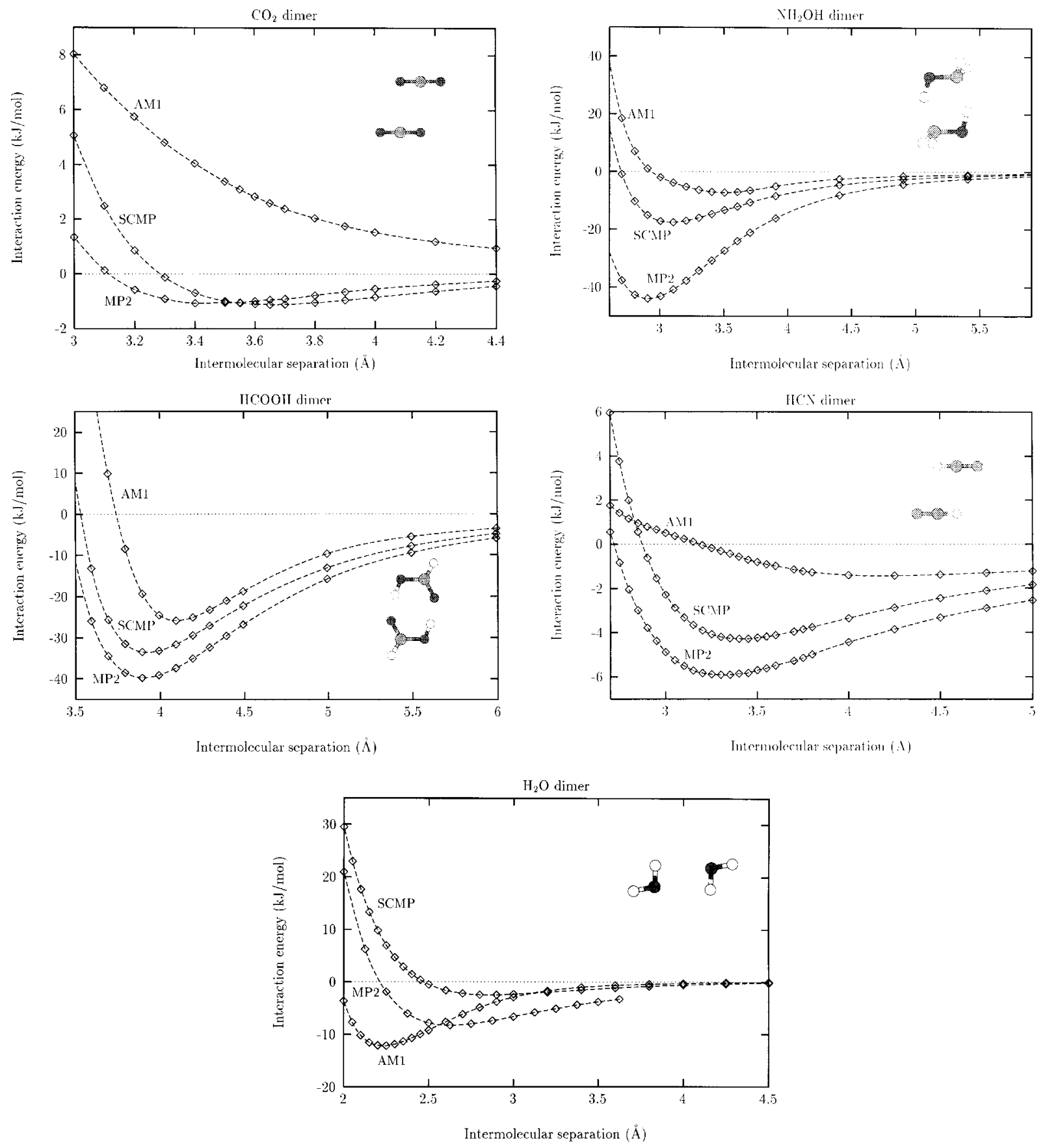

FIGURE 1. Interaction energies for a series of symmetrical dimers calculated by the usual AM1 model Hamiltonian, by BSSE-corrected $a b$ initio method at the MP2 level, using the correlation-consistent polarized valence double zeta (cc-pVDZ) basis set, and by the SCMP method, using the $\Delta E_{\mathrm{SCMP}}$ energy. The water dimer has been calculated with the aug-cc-pVDZ basis set. 
2. standard AM1 interaction energy (standard dimer calculation), and

3. basis-set superposition error corrected MP2 interaction energy with correlation-consistent polarized valence double $\zeta$ basis $(\mathrm{MP} 2 / \mathrm{cc}-$ $\mathrm{pVDZ}^{56}$ (standard dimer calculation).

The latter method was considered as a reference. Because $\Delta E^{\mathrm{SCMP}}$ is based on the AM1 method, a comparison of the first two potentials shows the improvement in the description of the intermolecular interactions as achieved by deriving $\Delta E^{\mathrm{SCMP}}$ from standard AM1.

Figure 1 shows that the $\Delta E^{\mathrm{SCMP}}$ potential curves are consistently closer to the MP2 results than the AM1 curves. In most of the systems the $\Delta E^{\mathrm{SCMP}}$ potential reproduces the intermolecular separation corresponding to the energy minimum of the reference potential within $0.1-0.2 \AA$ In the case of the very flat curve of the $\mathrm{CO}_{2}$ dimer, the difference is about $0.2 \AA$. The error in the energy at the minimum varies regarding both its absolute and relative value. The error is largest for the $\mathrm{NH}_{2} \mathrm{OH}$ dimer $(25 \mathrm{~kJ} / \mathrm{mol}$, over $50 \%)$ while in all other cases it is considerably lower.

With the exception of the water dimer, the AM1 interaction energy curves are higher than $\Delta E^{\mathrm{SCMP}}$ in almost the whole range studied. In all cases, $\Delta E^{\mathrm{SCMP}}$ reproduces the reference curve better than does AM1. The potential curves of Figure 1 support the idea that the direct use of semiempirical (intermotif) integrals is not appropriate for reproducing intermolecular interaction energies. The replacement of intermotif integrals by interaction of self-consistent (SC) multipoles and the addition of an appropriate dispersion-repulsion potential not only compensates for the error caused by the neglect of resonance and exchange integrals that are inherent in the SCMP approach, but it results in an intermolecular potential considerably superior to AM1.

The relatively good performance of the potential model obtained from the independently derived QM and classical dispersion-repulsion potentials suggests that not only the sum, but also the separate components are basically correct. This finding is in line with previous observations that NDDO semiempirical electrostatic potentials are able to reproduce more accurate $a b$ initio electrostatic potentials, primarily outside the van der Waals envelope where the short-range penetration component is less important. ${ }^{54}$

\section{Results and Discussion}

\section{LATTICE ENERGIES}

The reliability of the SCMP model has been studied for a series of molecular crystals (cf. Fig. 2) by comparing experimental sublimation enthalpies, with lattice energies calculated with various parameters. Due to the approximations involved when equating calculated lattice energies with experimental sublimation enthalpies, the intrinsic significance threshold of such a comparison has been estimated to be about $8.5 \mathrm{~kJ} / \mathrm{mol}^{57} \mathrm{~A}$ more stringent test of the model would be to check whether the theoretical minimum of the lattice energy is close enough to the experimental structure. Such a comparison is going to be done in the near future. ${ }^{55}$

The coordinates of atoms of a single molecule of the crystal as determined by X-ray or neutron diffraction measurements were taken and, follow-<smiles>c1ccc2[nH]cnc2c1</smiles>

BZDMAZ<smiles>CCOC(N)=O</smiles>

ECARBM01<smiles>O=C1C[CH]CO1</smiles><smiles>Nc1c([N+](=O)[O-])c(N)c([N+](=O)[O-])c(N)c1[N+](=O)[O-]</smiles>

TAT:NBZ<smiles>NC(N)=O</smiles>

UREAXX

FORMAM<smiles>O=CNC=O</smiles>

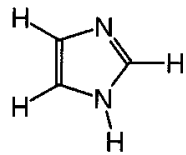

IMAZOL13<smiles>N#CN=C(N)N</smiles>

CYAMPDO:
SUCANH

O<smiles>Nc1cc[nH]c(=O)n1</smiles>

CYTSINO1<smiles>O=c1cc[nH]c(=O)[nH]1</smiles>

LRACIL
FIGURE 2. Structural formulae of the molecules. The CSD codens are listed in Table 1. 
ing the protocol of Willock et al., ${ }^{6}$ the $\mathrm{C}-\mathrm{H}$, $\mathrm{N}-\mathrm{H}$, and $\mathrm{O}-\mathrm{H}$ bond distances were set to 1.08 , 1.01, and $0.96 \AA$, respectively. Selected data concerning the crystal structures used in this study are compiled in Table $\mathrm{I}^{58-68}$

Then the wave function and the energy of the free molecule were calculated. The crystal SCF calculation was started with the density of the isolated molecule; thus, the difference between the energy in the first SCF cycle and that of the isolated molecule gives the electrostatic component $\left(E^{\text {ele }}\right)$ of the cohesion energy. The difference between the SCF energy and the energy calculated with the density matrix of the isolated molecule is the induction energy ( $E^{\text {ind }}$ ).

The electrostatic and induction energies, as calculated with various parameterizations, are presented in Table II. Considering the effect of param- eterization on the electrostatic energy, in most cases the order $E_{\mathrm{PM} 3}^{\mathrm{ele}}>E_{\mathrm{MNDO}}^{\mathrm{ele}}>E_{\mathrm{AM} 1}^{\mathrm{ele}}$ is found. The dependence of the induction energy on the parameterization is less significant. However, it should be noted that in the PM3 parameterization an artefactual minimum of the core repulsion function appears, which may produce unphysical distortions of the intermolecular potential energy surface. ${ }^{69,70}$ Due to this feature, the PM3 model is inappropriate for the present purpose. The AM1 method suffers much less from this problem. ${ }^{69}$

Table III presents calculated energies as obtained with AM1 parameters $\left(\Delta E^{\mathrm{SCMP}}\right)$ and experimental sublimation enthalpies $\left(E^{\exp }\right) .^{71-73}$ Taking into account that in most cases the calculated energies underestimate the experimental values, and that AM1 predicts the largest stabilization (cf. Table II), it is found that the best agreement between

TABLE I.

Cambridge Structural Database (CSD) Coden, Space Group, and Number Molecules / Unit Cell for Crystal Structures Considered.

\begin{tabular}{llll}
\hline \multicolumn{1}{c}{ Molecule } & CSD Coden & Space Group & $Z^{\text {a }}$ \\
\hline Imidazol $^{58}$ & IMAZOL13 & $P 2_{1} / c$ & 4 \\
Urea $^{59}$ & UREAXX & $P \overline{4} 2_{1} m$ & 2 \\
Benzimidazol $^{60}$ & BZDMAZ & $P 2_{1} n b$ & 4 \\
Formamide $^{61}$ & FORMAM & $P 2_{1} / c$ & 4 \\
Cytosine $^{62}$ & CYTSIN01 & $P 2_{1} 2_{1} 2_{1}$ & 4 \\
$1,3,5-$ Triamino-2,4,6-trinitro-benzene $^{3}$ & TATNBZ & $P \overline{1}$ & 2 \\
Uraci6 $^{64}$ & URACIL & $P 2_{1} / a$ & 4 \\
Maleic anhydride $^{65}$ & MLEICA & $P 2_{1} 2_{1} 2_{1}$ & 4 \\
Succinic anhydride $^{66}$ & SUCANH & $P 2_{1} 2_{1} 2_{1}$ & 4 \\
Urethane $^{67}$ & ECARBM01 & $P \overline{1}$ & 2 \\
Dicyanodiamide $^{68}$ & CYAMPD03 & $C 2 / c$ & 8 \\
\hline
\end{tabular}

${ }^{\mathrm{a}}$ Number of symmetry related molecules in the unit cell.

TABLE II.

Electrostatic and Induction Contributions (kJ / mol) to Stabilization Energy of Crystals as Calculated by MNDO, AM1, and PM3 Parameterizations.

\begin{tabular}{lcccrrr}
\hline \multicolumn{1}{c}{ Molecule } & $E_{\text {MNDO }}^{\text {ele }}$ & $E_{\text {AM1 }}^{\text {ele }}$ & $E_{\mathrm{PM} 3}^{\text {ele }}$ & $E_{\text {MNDO }}^{\text {ind }}$ & $E_{\text {AM1 }}^{\text {ind }}$ & $E_{\text {PM3 }}^{\text {ind }}$ \\
\hline Imidazol & -37.7 & -42.1 & -35.8 & -13.8 & -15.8 & -15.8 \\
Urea & -57.6 & -62.5 & -49.4 & -21.6 & -23.3 & -19.7 \\
Benzimidazol & -33.4 & -42.0 & -35.7 & -12.6 & -15.0 & -15.5 \\
Formamide & -40.9 & -44.3 & -36.5 & -15.0 & -16.4 & -16.0 \\
Cytosine & -74.1 & -79.7 & -67.9 & -24.8 & -26.9 & -25.8 \\
TATNBZ & -43.1 & -53.0 & -35.8 & -7.8 & -7.6 & -7.4 \\
Uracil & -54.3 & -61.6 & -50.7 & -19.1 & -20.8 & -19.5 \\
Maleic anhydride & -32.6 & -27.6 & -28.4 & -6.4 & -5.9 & -6.0 \\
Succinic anhydride & -37.0 & -33.4 & -34.8 & -7.8 & -7.6 & -8.0 \\
Urethane & -33.3 & -36.4 & -28.3 & -8.4 & -9.1 & -9.0 \\
Dicyanodiamide & -74.3 & -76.4 & -68.5 & -25.2 & -26.9 & -29.3 \\
\hline
\end{tabular}


TABLE III.

Comparison of Calculated and Experimental Lattice Energies (kJ/ mol).

\begin{tabular}{|c|c|c|c|c|}
\hline Molecule & $\Delta E^{\mathrm{SCMP}}$ & $E^{\text {cal a }}$ & $E^{\text {calb }}$ & $E^{\exp }$ \\
\hline Imidazol & -82.6 & -87.9 & -67.2 & $-83.0^{c, d}$ \\
\hline Urea & -96.7 & -106.8 & -77.5 & $-98.6^{c}$ \\
\hline Benzimidazol & -94.7 & & & $-102.2^{\mathrm{e}}$ \\
\hline Formamide & -68.3 & -85.0 & -61.8 & $-71.7^{c, f}$ \\
\hline Cytosine & -145.7 & -169.3 & -128.5 & $-155.0^{c}$ \\
\hline TATNBZ & -177.5 & -191.0 & -154.0 & $-168.2^{c}$ \\
\hline Uracil & -107.6 & -130.5 & -102.7 & $-131.0^{c}$ \\
\hline Maleic anhydride & -67.9 & & & $-68.1^{\mathrm{g}}$ \\
\hline Succinic anhydride & -73.8 & & & $-82.3^{\mathrm{g}}$ \\
\hline Urethane & -71.2 & & & $-80.1^{g}$ \\
\hline Dicyanodiamide & -137.7 & & & $-129.3^{g}$ \\
\hline
\end{tabular}

${ }^{a}$ Energies with nonoptimized parameters given by Willock et al. ${ }^{6}$

${ }^{b}$ Energies with nonoptimized parameters given by Coombes et al. as EST + 0.9DMA. ${ }^{7}$

${ }^{\mathrm{c}}$ Reference 71 .

${ }^{\mathrm{d}}$ Other available value: $-83.1 .{ }^{72}$

${ }^{\mathrm{e}}$ Reference 72.

${ }^{\dagger}$ Other available value: $-73.9 .{ }^{73}$

${ }^{\mathrm{g}}$ Reference 73.

calculated and experimental lattice energies is achieved by using AM1 parameterization. Therefore, from now on the calculated cohesion energies $\left(\Delta E^{\mathrm{SCMP}}\right)$ refer to those obtained with $\mathrm{AM} 1 \mathrm{pa}-$ rameters.

According to Table III the calculated lattice energies reproduce the experimental sublimation enthalpies within $10 \mathrm{~kJ} / \mathrm{mol}$. The only exception is uracil, for which the difference between the experimental and the calculated lattice energy is 23 $\mathrm{kJ} / \mathrm{mol}$. In almost all the cases the lattice energies calculated at the experimental structure are higher than the experimental heats of sublimation. Because optimization of the structural parameters always lowers the energy, calculated cohesion energies are thought to move toward the experimental ones during lattice relaxation.

It is worth comparing our results to those of two recent publications ${ }^{6,7}$ on the calculation of crystal structures and energies for polar molecular crystals (see Table III). Considering the work of Willock et al., ${ }^{6}$ the only difference between their model and ours is in the electrostatic-induction part of the energy expression: Willock et al. use an $a b$ initio distributed multipole set to represent the electrostatic effects and they do not consider any induction contribution explicitly.

Coombes et al. ${ }^{7}$ also use an $a b$ initio distributed multipole model for representing electrostatic effects. Their dispersion-repulsion potential is different from that used by Willock et $a .^{6}$ and in the present work and appears to predict less stabilization of the crystal by the dispersion-repulsion energy component. Coombes et al. ${ }^{7}$ presented several combinations of the distributed multipole and the dispersion-repulsion potentials and applied them to a wide range of molecular crystals. Their lattice energies in the experimental geometry calculated as the sum of the scaled DMA + dispersion-repulsion energy (designated by 0.9DMA + EST in ref. 7 and in Table III of the present contribution) consistently underestimate the experimental values. We note, that the DMA + EST model (not presented explicitly by the authors, but reconstituted from the published data in ref. 7), when applied at the experimental structure, reproduces the experimental values fairly well. The optimization of the structural parameters of the crystals considerably improved the agreement between the lattice energies predicted by the 0.9DMA + EST model and experimental values. The deviations between them are attributed by the authors to the incomplete inclusion of the induction and charge transfer components and to the uncertainty in the experimental lattice energies.

Comparing our results with the above cited two works, the overall agreement between calculated and experimental lattice energies is similar (see Table III). In contrast to those models, ours includes a semiempirical method to describe electrostatics. The computational requirements of the quantum chemical calculation shows the typical 
behavior of semiempirical methods. For small unit cells it is comparable to that of the empirical dispersion-repulsion energy that is almost instantaneous on modern workstations. Thus, molecular crystals with relatively large unit cells, containing 50-100 atoms, which is typical, for example, in pharmaceutical research can be routinely treated.

An important feature of our method is the explicit treatment of the induction energy. Data in Table III show that the polarization component may be as large as $1 / 3$ of the electrostatic term. This considerably exceeds the polarization component typical in binary interactions but seems to be in line with $a b$ initio SCMP results. ${ }^{37}$ The large magnitude of the induction energy points out that a correct description of the lattice energy of crystals requires the accurate reproduction of the induction energy component. The availability of separate electrostatic and induction energies offers the possibility to test the approach that uses a set of enhanced multipoles to implicitly include polarization in the electrostatic energy. Such an approach would be justified by a constant ratio of the two energy components. Comparing the electrostatic $\left(E_{\mathrm{AM} 1}^{\mathrm{ele}}\right)$ and induction $\left(E_{\mathrm{AM} 1}^{\mathrm{ind}}\right)$ energies of Table II, their ratio is indeed near 3 in most cases. There are, however, notable deviations from this rule; the most important is found for TATNBZ, where the induction energy is relatively small and $\left(E_{\mathrm{AM} 1}^{\mathrm{ele}}\right) /\left(E_{\mathrm{AM} 1}^{\text {ind }}\right)$ is as high as 7 .

At this point it should be noted that it is difficult to predict how much induction energy has been adsorbed into the dispersion-repulsion potential derived by Willock et al. $^{6}$ and applied in the present study. Its parameters were fitted to experimental lattice energies by using no explicit induction term and by using a distributed multipole set calculated from HF 6-31G** wave functions, which are known to overestimate the magnitude of the multipoles, therefore mimicking, at least to some extent, induction effects. As far as some of the potential energy parameters are estimated on an experimental basis, ambiguities concerning the relative importance of various interaction energy components cannot be fully eliminated.

Net atomic charges calculated in the SCMP approach are enhanced with respect to values of the isolated molecules. This enhancement affects primarily the H-bond acceptor atom. The dipole moments are also increased by factors between 1.2 and 1.6 (Table IV).
TABLE IV.

Dipole Moment of Free Molecules ( $\mu^{\mathrm{mol}}$ ) Compared to Those in Crystal Calculated by SCRF and SCMP Methods (Debye Units).

\begin{tabular}{lllr}
\hline \multicolumn{1}{c}{ Molecule } & $\mu^{\mathrm{mol}}$ & $\mu^{\text {SCRF }}$ & $\mu^{\text {SCMP }}$ \\
\hline Imidazol & 3.74 & 4.70 & 5.58 \\
Urea & 4.46 & 5.54 & 6.47 \\
Benzimidazol & 3.28 & 4.34 & 5.16 \\
Formamide & 3.94 & 4.84 & 5.65 \\
Cytosine & 6.51 & 8.68 & 9.15 \\
TATNBZ & 0.27 & 0.28 & 0.43 \\
Uracil & 4.47 & 5.85 & 6.73 \\
Maleic anhydride & 4.04 & 4.81 & 5.00 \\
Succinic anhydride & 4.66 & 5.69 & 5.77 \\
Urethane & 2.34 & 3.07 & 3.32 \\
Dicyanodiamide & 7.40 & 9.51 & 10.74 \\
\hline
\end{tabular}

The dielectric constant, $\varepsilon$, in the SCRF model has been chosen to be very high in order to approximate the best the in-crystal dipole moment.

\section{SCMP VERSUS SCRF MODELS}

The effect of the crystalline environment on the molecular charge distribution and/or geometry is sometimes taken into account in quantum chemical calculations by a simple dielectric continuum model. ${ }^{43}$ Previous analysis of both formalisms clearly showed ${ }^{48}$ that these two approaches, the SCMP and SCRF, lead to analogous quantum chemical equations. The main difference is in the evaluation of the effective response functions of the environment, specific to the model of the medium. Nevertheless, in both cases the influence of the medium is manifested by a global screening effect on the direct Coulomb interactions between real charges. In the SCMP model one appropriately takes into account the anisotropy of the crystal, while the continuum model corresponds usually to an isotropic environment.

It is possible to judge whether the SCRF approach is an appropriate model for crystals by comparing the relevant terms of the effective Madelung kernel and of the reaction potential response function. Alternatively, instead of the direct study of the response functions, we can examine observables, like the in-crystal dipole moment.

Our SCRF calculations were done with the ellipsoidal cavity model of Rinaldi et al., ${ }^{74-76}$ using a monocentered multipolar expansion of the direct and reaction potentials, as implemented in the Geomos semiempirical program package. ${ }^{77}$ The SCRF model was used with a sixth-order singlecentered multipole expansion of the molecular 
charge distribution, the size of the cavity was taken from standard prescriptions, and the dielectric permittivity, $\varepsilon$, was varied between 1 and 1000. These calculations indicated that the maximum enhancement of the molecular dipole moment in a continuous dielectric medium (without modifying the standard cavity size) is $0.7-2.1 \mathrm{D}$ (3-33\%). This enhancement is still 1-18\% less than the one calculated by the SCMP model of the crystalline environment.

\section{Conclusions}

We conclude that the computational approach, based on the implementation of the SCMP with a semiempirical NDDO model Hamiltonian, is an effective way to obtain lattice energies for molecular crystals. It is suggested that the AM1 Hamiltonian with atomic point multipoles can be successfully combined with the atom-atom dispersionrepulsion potential proposed by Willock et al. ${ }^{6}$ This method provides a realistic description of dissociation curves for various van der Waals dimer systems. Calculated lattice energies, composed of electrostatic, induction, and dispersionrepulsion components, are in reasonable agreement with available experimental sublimation enthalpies.

The good performance of the presented quantum classical model can be attributed to a reliable modeling of electrostatic + induction interactions using SC multipoles calculated by the SCMP method and to the application of an empirical dispersion-repulsion potential that does not adsorb the electrostatic contribution. Due to its low computational cost, the SCMP-NDDO approach is primarily suggested for molecular crystals with large asymmetric units. The implementation of SCMP-NDDO forces is in progress. ${ }^{55}$

We showed that the continuum model should be used only as a crude first approximation to estimate the influence of solid-state environments. Although the general trend of the condensed phase induction effects is the same, highly ordered crystalline environments may lead to much stronger dipole moment enhancement than the continuum cavity models. It is recommended, in general, to apply the SCMP model, which explicitly uses the available experimental structures and is capable of taking into account the anisotropic nature of intermolecular interactions in crystals.

\section{Acknowledgments}

The authors are grateful to Prof. N. Rivail for the continuous interest in this work. We wish to thank the French and Hungarian governments for financial support in the framework of the program Balaton (No. 52), as well as for the grants from the National Fund for Scientific Research, Hungary (OTKA/T19387 and OTKA/T22012).

\section{References}

1. A. J. Pertsin and A. I. Kitaigorodsky, The Atom-Atom Potential Method, Springer-Verlag, Berlin, 1987.

2. G. R. Desiraju, Crystal Engineering: The Design of Organic Solids, Elsevier, Amsterdam, 1989.

3. G. Filippini and A. Gavezzotti, Acta Crystallogr. B, 49, 868 (1993).

4. J. Voogd, J. L. Derissen, and F. B. van Duijneveldt, J. Am. Chem. Soc., 103, 7702 (1981).

5. Z. Berkowitch-Yellin and L. Leiserowitz, J. Am. Chem. Soc., 104, 4052 (1982).

6. D. J. Willock, S. L. Price, M. Leslie, and C. R. A. Catlow, J. Comput. Chem., 16, 628 (1995).

7. D. S. Coombes, S. L. Price, D. J. Willock, and M. Leslie, J. Phys. Chem., 100, 7352 (1996).

8. K. M. Merz, Jr., J. Comp. Chem., 13, 749 (1992).

9. C. Chipot, J. G. Ángyán, B. Maigret, and H. A. Scheraga, J. Phys. Chem., 97, 9788 (1993).

10. C. Chipot, J. G. Ángyán, G. G. Ferenczy, and H. A. Scheraga, J. Phys. Chem., 97, 6628 (1993).

11. S. L. Price and A. J. Stone, J. Chem. Soc. Faraday Trans., 88, 1755 (1992).

12. T. R. Stouch and D.E. Williams, J. Comp. Chem., 13, 622 (1992).

13. C. A. Reynolds, J. W. Essex, and W. G. Richards, J. Am. Chem. Soc., 114, 9075 (1992).

14. T. R. Stouch and D. E. Williams, J. Comp. Chem., 14, 858 (1993).

15. F. Colonna and E.M. Evleth, Chem. Phys. Lett., 212, 665 (1993).

16. J. G. Ángyán and C. Chipot, Int. J. Quantum Chem., 52, 17 (1994).

17. C. Chipot, J. G. Ángyán, B. Maigret, and H. A. Scheraga, J. Phys. Chem., 97, 9797 (1993).

18. C. Gatti, B. Silvi, and F. Colonna, Chem. Phys. Lett., 247, 135 (1995).

19. S. T. Howard, M. B. Hursthouse, C. W. Lehmann, P. R. Mallinson, and C. S. Frampton, J. Chem. Phys., 97, 5616 (1992).

20. R. M. Metzger and J. B. Torrance, J. Am. Chem. Soc., 107, 117 (1985). 
21. R. Dovesi, M. Causá, R. Orlando, C. Roetti, and V. R. Saunders, J. Chem. Phys., 92, 7402 (1990).

22. R. Car and M. Parrinello, Phys. Rev. Lett., 55, 2471 (1985).

23. M. C. Payne, M. P. Teter, D. C. Allan, T. A. Arias, and J. D. Joannopoulos, Rev. Mod. Phys., 64, 1045 (1992).

24. G. Kresse and J. Hafner, Phys. Rev. B, 48, 13115 (1993).

25. E. J. Meijer and M. Sprik, J. Chem. Phys., 105, 8684 (1996).

26. J. Bridet, S. Fliszár, S. Odiot, and R. Pick, Int. J. Quantum Chem., 24, 687 (1983).

27. L. Turi and J. J. Dannenberg, J. Phys. Chem., 96, 5819 (1992).

28. P. Popelier, A. T. H. Lenstra, C. van Alsenoy, and H. J. Geise, J. Am. Chem. Soc., 111, 5658 (1989).

29. A. Peeters, C. Van Alsenoy, A. T. H. Lenstra, and H. J. Geise, J. Mol. Struct. (Theochem.), 304, 101 (1994).

30. A. T. H. Lenstra, C. Van Alsenoy, K. Verhulst, and H. J. Geise, Acta Crystallogr. B, 50, 96 (1994).

31. W. A. Sokalski, P. B. Keegstra, S. Roszak, and J. J. Kaufman, Int. J. Quantum Chem.: Quantum Chem. Symp., 24, 51 (1990).

32. Z. Barandiarán, L. Pueyo, and F. Gómez Beltrán, J. Chem. Phys., 78, 4612 (1983).

33. S. Huzinaga, L. Seijo, Z. Barandiarán, and M. Klobukowski, J. Chem. Phys., 86, 2132 (1987).

34. Z. Barandiarán and L. Seijo, J. Chem. Phys., 89, 5739 (1988).

35. L. Seijo and Z. Barandiarán, J. Chem. Phys., 98, 4041 (1993).

36. M. Tsukada, J. Phys. Soc. Jpn., 49, 1183 (1980).

37. J. G. Ángyán and B. Silvi, J. Chem. Phys., 86, 6957 (1987).

38. J. Almlöf and U. Wahlgren, Theor. Chim. Acta, 28, 161 (1973).

39. J. Zyss and G. Berthier, J. Chem. Phys., 77, 3635 (1982).

40. I. Panas, Chem. Phys. Lett., 194, 239 (1992).

41. I. Panas, Acta Crystallogr. A, 49, 881 (1993).

42. J. Li, H-L. Liu, and J. Ladik, Chem. Phys. Lett., 230, 414 (1994).

43. H. Jiao and P. v. R. Schleyer, J. Am. Chem. Soc., 116, 7429 (1994).

44. A. Warshel and M. Levitt, J. Mol. Biol., 103, 227 (1976).

45. M. J. Field, P. A. Bash, and M. Karplus, J. Comput. Chem., 11, 700 (1990).

46. G. G. Ferenczy, J.-L. Rivail, P. R. Surján and G. Náray-Szabó, J. Comput. Chem., 13, 830 (1992).

47. J. G. Ángyán and B. Silvi, In Modelling of Molecular Structures and Properties, J.-L. Rivail, Ed., Elsevier, Amsterdam, 1990, p. 337.

48. J. G. Ángyán, J. Math. Chem., 10, 93 (1992).

49. M. J. S. Dewar and W. Thiel, J. Am. Chem. Soc., 99, 4899 (1977).

50. M. J. S. Dewar, E. G. Zoebish, E. F. Healy, and J. J. P. Stewart, J. Am. Chem. Soc., 107, 3902 (1985).
51. J. J. P. Stewart, J. Comput. Chem., 10, 209 (1989).

52. M. Catti, Acta Crystallogr. A, 34, 974 (1978).

53. A. Gavezzotti and G. Filippini, J. Phys. Chem., 98, 4831 (1994).

54. G. Náray-Szabó and G. G. Ferenczy, Chem. Rev., 95, 829 (1995).

55. G. G. Ferenczy and J. G. Ángyán, manuscript in preparation.

56. R. A. Kendall, Jr., T. H. Dunning, and R. J. Harrison, J. Chem. Phys., 96, 6796 (1992).

57. A. Gavezzotti and G. Filippini, in Theoretical Aspects and Computer Modeling of the Molecular Solid State, A. Gavezzotti, Ed., Wiley, New York, 1997, p. 97.

58. B. M. Craven, R. K. McMulllan, J. D. Bell, and H. C. Freeman, Acta Crystallogr. B, 33, 2585 (1977).

59. S. Swaminathan, B. M. Craven, and R. K. McMullan, Acta Crystallogr. B, 40, 300 (1984).

60. C. J. Dik-Edixhoven, H. Schenk, and H. van Dermeer, Cryst. Struct. Commun., 2, 23 (1973).

61. T. Ottersen, Acta Chem. Scand. A, 29, 939 (1975).

62. R. J. McClure and B. M. Craven, Acta Crystallogr. B, 29, 1234 (1973).

63. H. H. Cady and A. C. Larson, Acta Crystallogr., 18, 485 (1965).

64. R. F. Stewart, Acta Crystallogr., 23, 23 (1967).

65. R. E. Marsh, E. Ubell, and H. E. Wilcox, Acta Crystallogr., 15, 35 (1962).

66. M. Ehrenberg, Acta Crystallogr., 19, 698 (1965).

67. B. H. Bracher and R. W. H. Small, Acta Crystallogr., 23, 410 (1967).

68. F. L. Hirshfeld and H. Hope, Acta Crystallogr. B, 36, 406 (1980).

69. G. I. Csonka, J. Comput. Chem., 14, 895 (1993).

70. V. Buss, J. Messinger, and N. Heuser, QCPE Bull., 11, 5 (1991).

71. J. S. Chickos, In Molecular Structure and Energetics, vol. 2, J. F. Liebman and A. Greenberg, Eds., VCH, New York, 1987, p. 67, quoted in ref. 7.

72. P. Jiménez, M. V. Roux, C. Turrión, and F. Gomis, J. Chem. Thermodyn., 19, 985 (1987).

73. H. G. M. De Wit, J. C. Van Miltenburg, and C. G. De Kruif, J. Chem. Thermodyn., 15, 651 (1983).

74. D. Rinaldi and J.-L. Rivail, Theor. Chim. Acta., 32, 57 (1973).

75. J.-L. Rivail and D. Rinaldi, Chem. Phys., 18, 233 (1976).

76. D. Rinaldi, F. Ruiz-López, and J.-L. Rivail, J. Chem. Phys., 78, 834 (1983).

77. D. Rinaldi, P. E. Hoggan, and A. Cartier, GEOMOS: SemiEmpirical SCF System for Dealing with Solvent Effects and Solid Surface Adsorption, QCPE, Indiana University, Bloomington, IN, Program 584, 1989; QCPE Bull., 9, 128 (1989). 